\title{
样点邻域同构曲面约束的散乱点云法向估计
}

\author{
孙殿柱 ${ }^{1}$ 梁增凯 ${ }^{1}$ 薄志成 ${ }^{1}$ 李延瑞 ${ }^{2}$ 沈江华 ${ }^{1}$ \\ (1. 山东理工大学机械工程学院 淄博 255049 ; \\ 2. 西安交通大学机械工程学院 西安 710049)
}

\begin{abstract}
摘要 : 针对现有点云法向估计算法难以兼顾估计结果的精度与稳健性问题, 以局部采样区域同构曲面作为样点邻域点集所反 映曲面形状约束，提出一种散乱点云法向估计方法。该方法将目标样点的邻域点集作为局部样本进行曲面重建，获取插值于 采样点集并与采样表面拓扑同构的局部网格曲面; 对曲面局部区域高斯映射结果进行聚类分析, 获取目标样点的各向同性邻 域面 ; 基于面片的正则度以及面片至目标样点的测地距离, 确定目标样点各向同性邻域面片法向的加权均值 , 并将所得结果 作为目标样点的法向估计结果。试验结果表明, 该方法在点云数据信噪比为 $40 \mathrm{~dB}$ 的情况下可保证 $98 \%$ 以上样点法向估计偏 差在 $10^{\circ}$ 以内, 可稳健处理含有噪声以及采样不均匀等缺陷的散乱点云法向估计问题, 对于含尖锐特征的点云亦能准确估计 样点法向，且具有较高的计算效率。
\end{abstract}

关键词：样点邻域；拓扑同构 ; 法向估计 ; 局部重建 ; 高斯映射

中图分类号 : TP391

\section{An Estimation Method for Normal of Unorganized Point Cloud Based on Local Isomorphic Surface}

\author{
SUN Dianzhu ${ }^{1} \quad$ LIANG Zengkai $^{1} \quad$ BO Zhicheng $^{1} \quad$ LI Yanrui $^{2} \quad$ SHEN Jianghua $^{1}$ \\ (1. School of Mechanical Engineering, Shandong University of Technology, Zibo 255049; \\ 2. School of Mechanical Engineering, Xi' an Jiaotong University, Xi'an 710049)
}

\begin{abstract}
For solve the problem that the current algorithms for estimating normals of point cloud are difficult to achieve a trade-off between accuracy and robustness of estimation results, a method for normal estimation based on local isomorphic surface is proposed. In this method, a set of neighborhood points of the target sample point is taken as the local sample to reconstruct the surface, and the local mesh surface interpolated in the sampling point set and isomorphic with the sampling surface topology is obtained. The results of Gauss mapping in the local area are clustered to obtain the isotropic neighborhood of the target sample point. Based on the regularity of the patches and the geodesic distance from the patches to the target sample point, the weighted mean of the normal of the patches in the isotropic neighborhood of the target sample point is determined, and the result obtained is taken as the normal estimation result of the target sample point. The experimental results show that the normal estimation deviation of more than $98 \%$ points can be guaranteed to be less than $10^{\circ}$ under the condition of point cloud data SNR of $40 \mathrm{~dB}$. The proposed algorithm can robustly deal with the problem of normal estimation of scattered point clouds with noise and non-uniform sampling. It can also accurately estimate the normal of surface samples in sharp feature areas and has high computational efficiency.
\end{abstract}

Key words : neighborhood of sample point ; topological isomorphism ; normal estimation ; local reconstruction ; Gaussian mapping

\section{0 前言}

点云数据具有简洁、灵活且无需保持拓扑一致 等特性，在工业制造、文物保护及医学等领域得到

* 国家自然科学基金(51575326)和山东省自然科学基金(ZR2015EM031)资 助项目。20181210 收到初稿, 20190530 收到修改稿
广泛应用。法向作为点云的重要属性之一，其计算 结果的准确性直接影响点云数据后期处理效果, 因 此点云的法向估计问题长期以来深受关注 ${ }^{[1-2]}$ 。

目前法向估计主要分为依据点云全局信息的方 法和基于局部样本的方法两类。AMENTA 等 ${ }^{[3-5]}$ 基 于点云 Delaunay 网格剖分及对偶 Voronoi 图建立数 据间拓扑关系, 计算任一样点在其 Voronoi 单元中 
的极点, 将极点与样点的连线作为该样点法向。该 方法参考点云全局信息，对尖锐特征区域数据具有 较好的估计效果，但易受噪声干扰。文献[6]算法将 Voronoi 单元格极点扩展到大 Delaunay 球来估计点 云法向，虽然一定程度上可减弱噪声干扰，但其基 于全局 Delaunay 网格剖分时空复杂度较高, 难以适 用于大规模点云数据的处理。HOPPE 等 ${ }^{[7]}$ 采用局部 表面拟合的方法 , 在假定采样表面为光滑流形的前 提下, 对样点局部 $k$ 邻域点集进行平面拟合, 以所 得平面的法向作为样点法向。局部表面拟合的方法 原理简单, 但计算精度较低, 当点云采样不均匀或 模型结构复杂时, 其法向估计结果往往误差较大。 在此基础上文献[8-10]等通过优化局部拟合表面提 高了法向估计的计算精度和稳健性, 优化过程通常 基于更为复杂的表面构建方法或基于产生大量候选 解的复杂抽样过程, 导致时间复杂度较高或需要大 量调整参数。其中, 文献[8]算法结合异常值诊断与 主成分分析方法, 对剔除离群点的局部样本进行主 成分分析进而估计样点法向, 虽然可以提高法向估 计的稳健性, 但在尖锐特征区域样点法向难以准确 估计。文献[11]对局部样本进行泊松曲面重建, 虽 然拟合精度较高, 但过程繁琐。CHEN 等 ${ }^{[12]}$ 基于网 格曲面将顶点的法向定义为邻域网格面片法向的加 权和，依据权重因子的不同产生了诸多变体 ${ }^{[13-14]}$ 。 基于邻域网格的方法可较好反映曲面局部属性, 快 速准确地实现样点法向估计, 但该类方法以将点云 转化为离散网格为前提, 邻域网格拓扑正确与否影 响着样点法向估计精度。

在实际扫描原表面获取点云过程中，因测量距 离及扫描角度的变化即便对于光滑曲面仍会存在采 样误差, 特别当点云模型坐标出现法向偏移误差时, 样点法向估计结果往往精度较低, 且在尖锐特征区 域法向估计结果偏差较大。为解决这一问题, 本文 将目标样点的邻域点集作为局部样本进行曲面重 建，获得插值于采样点集且与原表面拓扑同构的局 部网格曲面, 通过对局部网格曲面进行高斯映射获 取样点的各向同性邻域面, 基于各向同性邻域面的 正则度及邻域面到目标样点的测地距离进行样点 法向的统计推断。试验结果表明, 该方法可有效抑 制曲面采样误差的干扰, 实现尖锐特征区域样点法 向准确估计, 且具有较高的计算效率。

\section{1 局部拓扑重建}

为获得插值于采样点集的高质量 Delaunay 三 角网格曲面, 本文依据局部平坦程度, 采用二维
Delaunay 网格剖分与三维 Delaunay 网格过滤相结合 的策略对局部样本进行重建。对于采样点集 $S$ 中目 标样点 $p$, 将 $p$ 的 $k$ 邻域点集作为局部重建样本 $\lambda(p)$ ，通过适度扩大邻域搜索范围对该样本添加辅 助点。设 $k_{\eta}$ 为获取 $\lambda(p)$ 时所需的邻域样点数量, $k_{\zeta}$ 为添加辅助点后邻域点集样点数量, 局部重建算法 的具体流程如下。

(1) 搜索 $p$ 的 $k_{\eta}$ 邻域点集, 获得局部重建样本 $\lambda(p)$ 。

(2) 搜索 $p$ 的 $k_{\zeta}$ 邻域点集，获得包含辅助点的 局部重建样本 $\lambda(\tilde{p})$, 在 $\lambda(\tilde{p})$ 中若 $\exists p_{i}, p_{i} \in \lambda(\tilde{p})$ 且 $p_{i} \notin \lambda(p)$ ，则将 $p_{i}$ 标记为辅助点。

(3) 对 $\lambda(\tilde{p})$ 中样点进行共面检测，若共面，则 对 $\lambda(\tilde{p})$ 进行二维 Delaunay 网格剖分, 输出剖分的 局部重建网格, 程序结束。

(4) 对 $\lambda(\tilde{p})$ 进行三维 Delaunay 网格剖分, 获得 四面体集合 $D(p)$, 并求出对应的 Voronoi 图 $V(p)$ 。

(5) 对 $D(p)$ 中任一面片 $T_{i}$ 进行夹角检测，若检 测未通过, 则将 $T_{i}$ 从 $D(p)$ 中删除, 否则保留。

(6) 对 $D(p)$ 进行流形提取[15], 输出局部重建 网格, 程序结束。

在上述步骤(5)中，对 $T_{i}$ 进行夹角检测的具体流 程如下。

(1) 提取面片 $T_{i}$ 的顶点 $\left\{v_{1}, v_{2}, v_{3}\right\}$ 。

(2) 检测每个顶点的标记, 若三个顶点中存在 辅助点, 则该面片未通过检测，程序结束。

(3) 获取面片 $T_{i}$ 对偶 Voronoi 边的端点 $l_{1} 、 l_{2}$ 。

(4) 对于顶点 $v_{1}$, 计算 Voronoi 单元中与其距离 最远的 Voronoi 顶点, 并将由 $v_{1}$ 指向该顶点的向量 记为 $\boldsymbol{v}_{v_{1}}$ 。

(5) 计算 $\boldsymbol{v}_{1} \boldsymbol{l}_{1}$ 和 $\boldsymbol{v}_{1} \boldsymbol{l}_{2}$ 与 $\boldsymbol{v}_{v_{1}}$ 的夹角 $\theta_{1}$ 和 $\theta_{2}$, 其中 $\theta_{1}=\angle\left(\boldsymbol{v}_{1} \mathbf{l}_{1}, \boldsymbol{v}_{v_{1}}\right) ， \theta_{2}=\angle\left(\boldsymbol{v}_{1} \boldsymbol{l}_{2}, \boldsymbol{v}_{v_{1}}\right)$ 。若 $\theta_{1}$ 与 $\theta_{2}$ 均小于 $\pi / 8$ 或均大于 $7 \pi / 8$, 则该面片未通过检测。对 $v_{2}$ 和 $v_{3}$ 进行相同操作。

局部样本 $\lambda(p)$ 的重建结果如图 1 所示, 在平坦 区域和高曲率区域均可获得拓扑正确的局部重建结 果，可准确反映曲面局部形状。

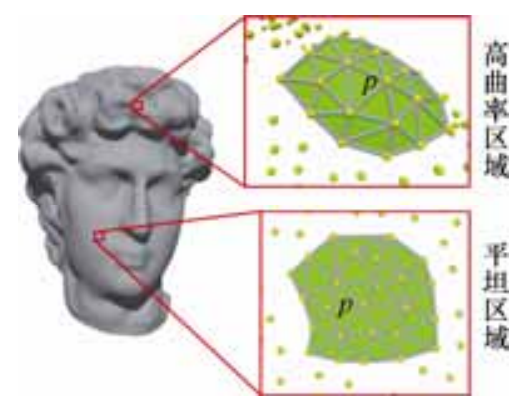

图 1 曲面局部重建结果 


\section{2 顶点邻域形心权重}

由局部曲面重建获得的 Delaunay 网格曲面包 含着样点之间的拓扑关系, 可基于网格顶点的邻接 信息计算样点法向。对网格曲面上任一顶点, 可将 其邻域面法向的加权和作为该顶点法向的估计结 果, 如图 2 所示。在分析离散网格顶点邻域面几何 特性的基础上，基于顶点邻域面的形状以及邻域面 质心到该顶点的测地距离确定邻域面法向的权重 值，使网格顶点法向计算更为稳定与精确。

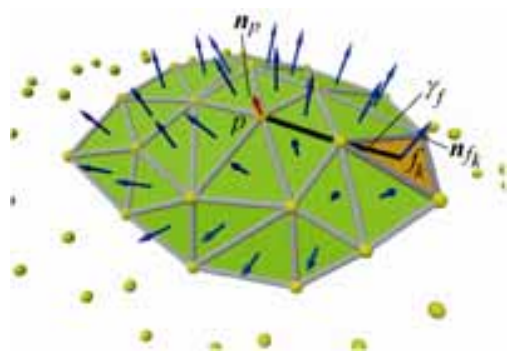

图 2 顶点法向估计

网格曲面上顶点法向计算公式可表示为

$$
\boldsymbol{n}_{p}=\sum_{k=1}^{m} c_{k} \boldsymbol{n}_{f_{k}} /\left\|\sum_{k=1}^{m} c_{k} \boldsymbol{n}_{f_{k}}\right\|
$$

式中, $m$ 为顶点邻域面片的数量 ; $c_{k}$ 为邻域面片 $f_{k}$ 的权重值 ; $\boldsymbol{n}_{f_{k}}$ 表示邻域面片 $f_{k}$ 的法向。

三角网格曲面以三角面片形状均为等边三角形 为质量最优 ${ }^{[16]}$ 。三角面片形状接近等边三角形的程 度以三角面片正则度表示, 三角面片正则度越高, 其形状越接近等边三角形。在同等条件下, 顶点邻 域三角面片正则度越高，该顶点处局部性质计算越 稳定, 顶点法向估计精度越高。为量化三角面片的 正则度, 计算三角面片外接圆圆心与其内切圆圆心 的距离并分析该距离与三角面片正则度之间的关 系。根据欧拉定理, 三角形外接圆圆心与其内切圆 圆心的距离 $d_{c}$ 可表示为

$$
d_{c}=\sqrt{R^{2}-2 R r}
$$

式中， $R$ 为三角形外接圆半径 ; $r$ 为三角形内切圆 半径。 $d_{c}$ 值越小, 三角形越接近等边三角形, 正则 度越高。考虑到三角形 $d_{c}$ 值的大小会受到三角形尺 寸的影响，直接将其作为三角面片正则度的度量标 准不够准确, 可将其与三角形外接圆半径的比值作 为三角面片正则度的度量依据。因此，三角面片 $f$ 的 正则度 $R(f)$ 可表示为

$$
R(f)=1-\frac{d_{c_{f}}}{R_{f}}=\frac{R_{f}-\sqrt{R_{f}^{2}-2 R_{f} r_{f}}}{R_{f}}
$$

式中， $d_{c_{f}}$ 表示 $f$ 的外接圆圆心到其内切圆圆心的 距离; $R_{f} 、 r_{f}$ 分别为 $f$ 的外接圆半径和内切圆半径。 随着 $f$ 的 $d_{c}$ 值递减， $R(f)$ 在 $[0,1]$ 区间递增。在 $[0,1]$ 区间内，随着 $R(f)$ 的增大，三角面片的正则度越高， 其法向对顶点法向的贡献越大。当 $f$ 形状为退化三 角形，即三点共线时，其值为 0 , 可认为该面片法 向对顶点法向没有影响; 当 $f$ 形状为等边三角形时， $R(f)$ 值为 1 , 则该面片法向对顶点的法向估计的影 响最大。

在顶点邻域面片正则度相同的情况下，邻域面 片与顶点的邻近程度也影响着顶点法向估计结果的 准确性。邻近程度越高的邻域面片，其几何性质与 顶点处相似度越高，对顶点法向估计的影响越大。 由于测地线表示曲面中两点的局域最短路径，根据 测地线的长度即测地距离可度量曲面中两点的远近 程度。因此, 可基于网格曲面中顶点到其邻域面片 质心的测地距离度量邻域面片与顶点的邻近程度。 为方便计算，利用 Dijkstra 算法 ${ }^{[17]}$ 获取局部网格曲 面中从顶点到其邻域面片质心的最短路径，近似为 两点间的测地线。

获得顶点至其邻域面 $f$ 质心的近似测地线 $\gamma_{f}$ 后，计算该测地线的长度即为顶点至其邻域面的测 地距离 $d_{\gamma_{f}}$, 并利用高斯核函数 ${ }^{[18]}$ 计算该测地距离 下邻域面法向对顶点法向的权重 $g(f)$

$$
g(f)=G\left(\left\|\frac{d_{\gamma_{f}}}{d_{m}}\right\|^{2}\right)
$$

式中， $d_{m}$ 为顶点到其邻域面的最大测地距离。

基于顶点邻域面的正则度以及顶点到其邻域面 质心的测地距离可确定邻域面法向对顶点法向的权 重值，称其为形心权重，表示为

$$
c(f)=R(f) \cdot g(f)=\frac{R_{f}-\sqrt{R_{f}^{2}-2 R_{f} r_{f}}}{R_{f}} \cdot G\left(\left\|\frac{d_{\gamma_{f}}}{d_{m}}\right\|^{2}\right)
$$

顶点邻域的形心权重综合考虑了邻域面片形 状、尺寸以及与顶点的邻近程度对顶点法向的影响， 表达的权重值更加合理、准确。

\section{3 样点法向统计推断}

在获得局部 Delaunay 网格曲面后,基于顶点邻 域形心权重将目标样点邻域面片法向的加权和作为 目标样点的法向估计结果，可降低采样误差对样点 法向估计造成的影响，实现点云法向稳健估计。在 尖锐特征(如棱边、尖角)处的样点，其邻域面片可 
能来自多个采样表面，称为样点的各向异性邻域面， 若基于样点各向异性邻域面进行样点法向统计推 断, 会导致法向计算误差较大。因此, 在基于顶点 邻域面法向计算样点法向时, 应尽可能地选择位于 同一采样表面上的邻域面片，即样点的各向同性邻 域面，以提高样点法向的计算精度。

为兼顾样点法向估计的精度与稳健性, 以目标 样点 $p$ 的二阶邻域面作为参与目标样点法向计算的 候选面片集 $\Omega(p)=\left\{f_{i}\right\}, i=1,2,3, \cdots, m$ ，从中选取 样点的各向同性邻域面 $\Psi(p)=\left\{f_{j}\right\} \subseteq \Omega(p)$ 进行样 点 $p$ 法向的统计推断。

为准确获取 $\Psi(p)$ ，对 $\Omega(p)$ 中的各面片 $f_{i}$ 进 行高斯映射, 即将面片 $f_{i}$ 的单位化法向始端平移到 球心位于坐标原点的单位球球心处, 法向末端与球 面交于一点 $q_{i}$, 使 $f_{i}$ 与 $q_{i}$ 对应。 $\Omega(p)$ 中面片在单 位球上的像 $G(p)=\left\{q_{i}\right\}$ 称为 $\Omega(p)$ 的高斯图, 该单 位球称为高斯球。图 3 展示了 3 种典型区域样点邻 域面的高斯映射结果。若 $p$ 为光滑区域样点, 其邻 域各面片法向连续变化, $G(p)$ 表现为位于高斯球 面上的一点或密集分布的点簇; 若 $p$ 为棱边、尖角 等特征区域样点, 其邻域面为各向异性邻域面, 位 于不同表面的面片法向会有较大的偏差, $G(p)$ 表 现为高斯球面上两个或多个独立的点簇, 每个点簇 对应于不同采样表面上的面片。鉴于样点各向同性 邻域面的高斯图呈密集分布的点簇状, 可计算 $\Omega(p)$ 高斯图中点到其均值点的欧氏距离, 以其最 大距离是否大于给定阈值 $\varepsilon$ 为判断 $\Omega(p)$ 中是否包 含特征区域邻域面的标准。若最大距离小于國值 $\varepsilon$, 则 $\Omega(p)$ 为样点的各向同性邻域面, $\Psi(p)=\Omega(p)$; 反之, $\Omega(p)$ 中包含特征区域的面片，需对 $\Omega(p)$ 中 的面片进一步识别以获得 $\Psi(p)$ 。鉴于包含特征区 域面片 $\Omega(p)$ 的高斯图呈多个独立点簇状, 可基于 聚类 ${ }^{[19]}$ 的思想提取 $\Omega(p)$ 高斯图中 $\Psi(p)$ 的对应点 簇, 进而实现 $\Psi(p)$ 的获取。由于只需获得 $\Psi(p)$ 这 一类的数据, 所以只需对 $\Psi(p)$ 对应点簇进行聚类 即可。经过高斯映射后两面片法向的夹角映射为高 斯球面上两映射点之间的测地距离, 为便于计算以 映射点之间的欧氏距离作为两点的相似性度量。从 包含特征区域面片的 $\Omega(p)$ 中提取的 $\Psi(p)$ 的问题 可表示为在 $\Omega(p)$ 的高斯图 $G(p)$ 中寻找点集 $\Phi(p)=\left\{q_{j}\right\}$ 使得式(6)成立。

$$
\forall q_{j} \in \Phi(p),\left\|q_{j}-o_{p}\right\|<\varepsilon
$$

式中， $o_{p}$ 为聚类中心。 $\Phi(p)$ 对应的面片集即为 $\Psi(p)$ 。通过对 $\Omega(p)$ 高斯图聚类获取 $\Psi(p)$ 的关键 在于聚类中心的确定, 选择不同的聚类中心可能会
导致选取的 $\Psi(p)$ 位于不同的曲面上。为此, 对目 标样点一阶邻域面进行高斯映射，计算一阶邻域面 高斯图中各点的均值点, 以高斯图中距离均值点最 近的点作为聚类中心。

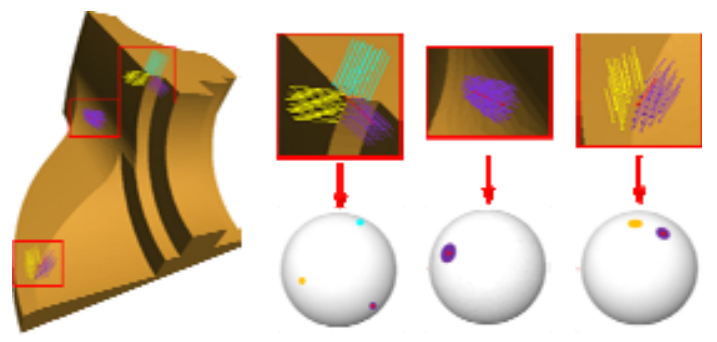

图 3 不同区域处样点邻域面的高斯图

在获取目标样点的各向同性邻域面 $\Psi(p)$ 后, 计算 $\Psi(p)$ 各面片法向及其形心权重, 以 $\Psi(p)$ 中各 面片法向的加权和作为目标样点 $p$ 的法向估计结 果，表示为

$$
\boldsymbol{n}_{p}=\sum_{f \in \Psi(p)} c_{f} \boldsymbol{n}_{f} /\left\|\sum_{f \in \Psi(p)} c_{f} \boldsymbol{n}_{f}\right\|
$$

综上所述, 对 $S$ 中任一样点 $p$, 其法向估计过 程如下。

(1) 获取 $p$ 的邻域点集并进行局部重建，获得 局部重建网格 $D(p)$ 。

(2) 从 $D(p)$ 中获取 $p$ 的二阶邻域面 $\Omega(p)$ ，对 $\Omega(p)$ 进行高斯映射，通过对映射结果进行聚类分 析得到 $p$ 的各向同性邻域面 $\Psi(p)$ 。

(3) 计算 $\Psi(p)$ 中各面片的法向以及形心权重。

(4) 按公式(7)计算 $p$ 的法向。

当对 $S$ 中所有样点遍历结束后, 利用文献[5]中 算法进行法向一致化处理。

\section{4 实验与分析}

在硬件配置为 HP xw8600 Workstation $(2.5 \mathrm{GHz}$, $4.0 \mathrm{~GB}$ 内存), 操作系统为 GNU/Linux 的环境下 利用文献[6]算法、文献[8]算法与本文算法对具有 代表性的不同点云模型进行测试。鉴于 $\mathrm{R}^{*}$ 树 ${ }^{[20]}$ 采用结点最小包围盒组织数据对象的空间邻近关 系, 对多维数据具有良好的空间索引性能, 本文 将其作为散乱点云的空间索引，实现邻域点集的 快速查找。

为验证本文算法法向估计的有效性，利用文献 [6]算法、文献[8]与本文算法对如图 4a 所示含有尖 锐特征区域的刀具模型点云数据进行法向估计。如 图 4b 所示文献[8]算法在尖锐特征区域法向估计效 果较差, 如图 4c 和图 4d 所示文献[6]算法与本文算 
法都具有较好的法向估计效果, 并且本文算法计算 所得法向在尖锐特征区域更为准确。

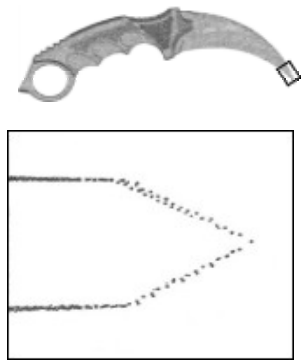

(a) 点云数据

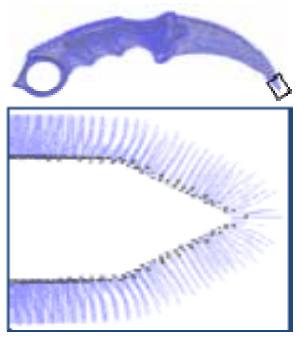

(c) 文献[6]算法

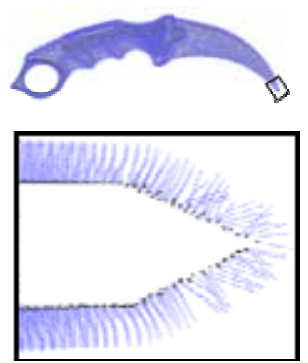

(b) 文献[8]算法

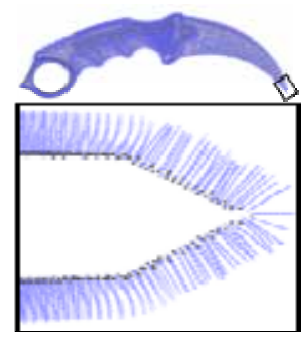

(d) 本文算法
图 4 刀具模型点云数据与不同算法法向估计结果

为定量分析本文算法的样点法向的计算精度， 统计计算法向与标准法向的偏差, 以法向偏差均方 根作为法向计算精度的统计量, 记为 $\theta_{\text {RMS }}$

$$
\begin{gathered}
\theta_{\mathrm{RMS}}=\sqrt{\frac{1}{|S|} \sum_{p \in S} f\left(\left\langle\boldsymbol{n}_{p r}, \boldsymbol{n}_{p e}\right\rangle\right)} \\
f\left(\left\langle\boldsymbol{n}_{p r}, \boldsymbol{n}_{p e}\right\rangle\right)= \begin{cases}\left\langle\boldsymbol{n}_{p r}, \boldsymbol{n}_{p e}\right\rangle, & \left\langle\boldsymbol{n}_{p r}, \boldsymbol{n}_{p e}\right\rangle<\tau \\
\frac{\pi}{2} & \text { 其他 }\end{cases}
\end{gathered}
$$

式中, $|S|$ 为采样点集 $S$ 中的样点数量; $\boldsymbol{n}_{p r} 、 \boldsymbol{n}_{p e}$ 分 别为样点的标准法向和计算法向 ; $\tau$ 为角度阈值， 一般取 $\pi / 18$ 。 $\theta_{\text {RMS }}$ 值越大, 计算法向误差越大, 精 度越低。为分析本文算法样点法向估计的稳健性, 利用 Matlab 向点云模型中加入不同程度的高斯白 噪声, 统计计算法向和标准法向的偏差, 偏差大于 $10^{\circ}$ 时称该样点为坏点, 统计不同噪声程度下坏点数 量(Bad point number, $\left.B_{\mathrm{PN}}\right)$ 。加入噪声后, 坏点数量 越少, 说明算法稳健性越好。

因复杂曲面点云数据难以获取标准法向，所以 利用 Matlab 构造规则点云模型，根据其样点坐标 可计算标准法向。实验采用 Mathlab 合成的圆柱 体点云模型, 其样点数量为 51883 , 向圆柱体点云 模型中分别加入信噪比 $S_{\mathrm{NR}}$ 为 $60 \mathrm{~dB} 、 50 \mathrm{~dB}$ 和 40 $\mathrm{dB}$ 的高斯白噪声，统计文献[6]算法、文献[8]算法 与本文算法在不同噪声情况下的 $\theta_{\mathrm{RMS}}$ 和 $B_{\mathrm{PN}}$, 如表 1 所示, 在无噪声条件下基于 Voronoi/Delaunay 的 文献[6]算法计算精度最高，本文算法次之，文献[8]
算法计算精度最低。文献[6]算法对噪声最为敏感， 随着噪声的增多, $B_{\mathrm{PN}}$ 和 $\theta_{\mathrm{RMS}}$ 值增长最为迅速 ; 基 于鲁棒主成分分析的文献[8]算法对噪声具有一定 的抵抗性, $B_{\mathrm{PN}}$ 和 $\theta_{\mathrm{RMS}}$ 值增长缓慢，但仍高于相应 条件下的文献[8]算法和本文算法的统计值 ; 随着 噪声程度的增大, 本文算法 $B_{\mathrm{PN}}$ 和 $\theta_{\mathrm{RMS}}$ 值最小且增 长速度介于文献[8]算法和文献[6]算法之间，这是 因为局部重建时会受噪声的影响, 但经提取样点各 向异性邻域以及面片法向加权处理后，一定程度上 降低了噪声的干扰， $S_{\mathrm{NR}}$ 为 $40 \mathrm{~dB}$ 时，仍有 $98 \%$ 以 上的样点法向估计结果偏差控制在 $10^{\circ}$ 以内。图 5 反映了 $S_{\mathrm{NR}}$ 为 $40 \mathrm{~dB}$ 的情况下 3 种算法法向估计结 果坏点的分布情况, 图中深黑色点表示坏点。如图 $5 b$ 所示文献[6]算法的坏点主要分布于两底面与侧 面的交界处以及圆柱体两底面上,除此之外在侧面 中也有一些坏点分布，文献[8]算法相比文献[6]算 法侧面上坏点的数量较少, 主要集中在两底面与侧 面的交界附近，这是因为文献[6]算法比文献[8]算 法对噪声较敏感, 当光滑的侧面上出现噪声时法向 计算结果受噪声干扰坏点较多。如图 $5 \mathrm{~d}$ 所示本文 算法坏点数量明显较少, 主要分布于侧面与两底 面的交界处, 这是因为存在噪声时, 获取样点的 各向同性邻域的准确度较低, 造成法向估计结果 偏差较大。

\begin{tabular}{|c|c|c|c|c|c|c|}
\hline \multirow{2}{*}{$S_{\mathrm{NR}} / \mathrm{dB}$} & \multicolumn{2}{|c|}{ 文献[6]算法 } & \multicolumn{2}{|c|}{ 文献[8]算法 } & \multicolumn{2}{|c|}{ 本文算法 } \\
\hline & $B_{\mathrm{PN}}$ & $\theta_{\mathrm{RMS}}$ & $B_{\mathrm{PN}}$ & $\theta_{\mathrm{RMS}}$ & $B_{\mathrm{PN}}$ & $\theta_{\mathrm{RMS}}$ \\
\hline 无噪声 & 22 & 1.001699 & 1207 & 1.025770 & 30 & 1.001722 \\
\hline 60 & 205 & 1.003363 & 1220 & 1.025919 & 169 & 1.003244 \\
\hline 50 & 332 & 1.005209 & 1245 & 1.026301 & 260 & 1.004919 \\
\hline 40 & 1198 & 1.018101 & 1430 & 1.026489 & 731 & 1.015129 \\
\hline
\end{tabular}

表 13 种方法下圆柱体模型的 $\boldsymbol{B}_{\mathrm{PN}}$ 和 $\theta_{\mathrm{RMS}}$ 比较

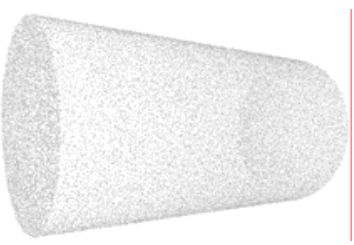

(a) $S_{\mathrm{NR}}$ 为 $40 \mathrm{~dB}$ 的圆柱体点云

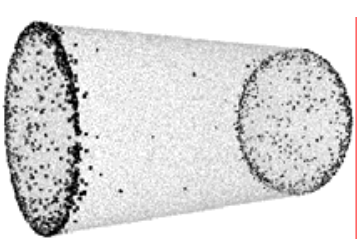

(c) 文献[8]算法

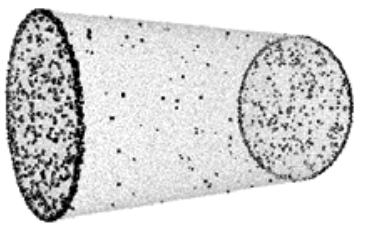

(b) 文献[6]算法

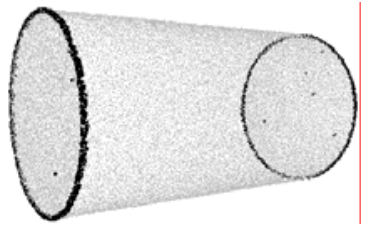

(d) 本文算法
图 $5 S_{\mathrm{NR}}$ 为 $40 \mathrm{~dB}$ 的圆柱体点云及各算法法向估计坏点分布 如图 6a 所示叶盘模型, 样点分布不均匀, 存在 一定的采样误差，基于 3 种算法所得法向结果利用 
文献[21]算法识别该模型中特征样点, 依据识别效 果判断法向估计结果的准确性。如图 $6 \mathrm{~b}$ 所示, 利用 文献[8]算法所得法向识别效果较差, 尤其在样点分 布不均匀区域, 样点识别准确性较低。在图 6c中, 由于文献[6]算法易受采样误差的干扰，法向估计结 果存在偏差, 导致过多的点被误判为特征点。从图 $6 \mathrm{~d}$ 可知，依据本文算法所得法向可较为准确地识别 出该模型中特征样点。

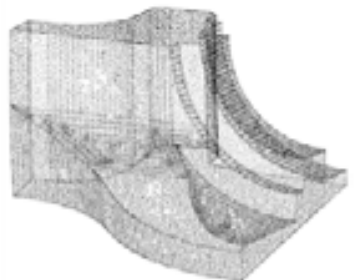

(a) 点云数据

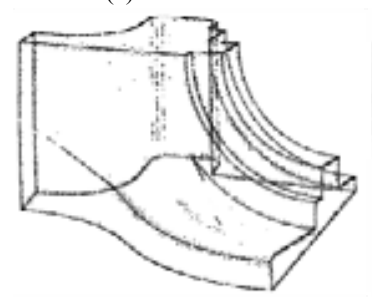

(c) 文献[6]算法

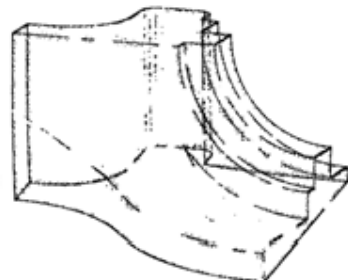

(b) 文献[8]算法

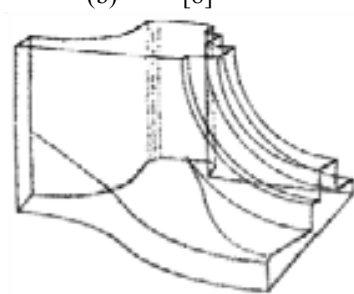

(d) 本文算法
图 6 叶盘点云数据与基于不同算法所得法向的 特征识别结果

为验证本文算法的运行效率，分别采用文献 [8]、文献[6]和本文算法对 6 组不同采样数据进行法 向估计, 实验结果如图 7 所示。本文算法采用局部 Delaunay 网格剖分的方式，运行效率略低于文献[8] 算法，但明显优于基于全局 Delaunay 网格剖分的文 献[6]算法, 点云样点数量为两百万个甚至更多时, 相比于文献[6]算法，本文算法所用时间可减少 30\% 以上。

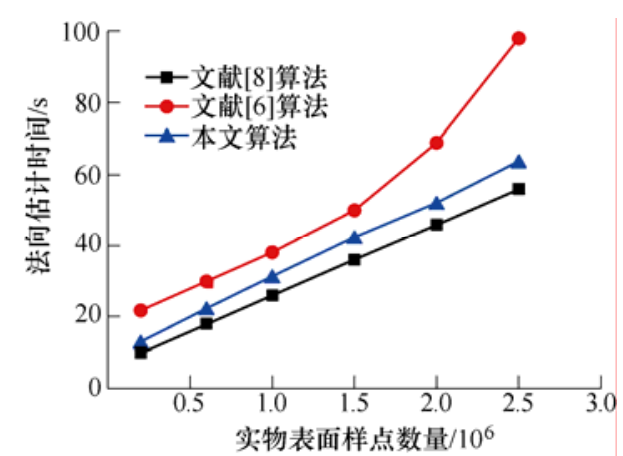

图 7 不同算法法向估计时间对比

本文算法所需主要参数有 $k_{\eta} 、 k_{\zeta}$ 和 $\varepsilon$ 。经多次 试验确定，当点云数据采样较均匀时，建议 $k_{\eta} 、 k_{\zeta}$ 取值为 15 和 25 , 当点云采样分布不均或采样误差 较大时, 可适当增大取值范围，建议 $k_{\eta} 、 k_{\zeta}$ 取值为
25 和 40 。 $\varepsilon$ 为提取样点各向同性邻域面的阈值, 取 较小的 $\varepsilon$ 值可提高样点各向同性邻域面获取的准确 度，但同时它也可能错误地将位于平滑区域中的样 点识别为特征样点, 导致算法稳健性降低; 取较大 的 $\varepsilon$ 值可充分利用样点的邻域面进行样点法向估 计，但易造成尖锐特征处样点法向估计不准确。建 议 $\varepsilon$ 的取值范围为 $0.15 \sim 0.25$ 。

\section{5 结论}

(1) 通过局部重建所得局部样本约束曲面插值 于采样点集且与原表面拓扑同构，可准确反映局部 曲面的拓扑结构，为保证基于局部曲面进行点云法 向准确估计奠定了基础。在此基础上, 基于网格面 片的正则度以及邻域面质心到顶点的测地距离对顶 点法向估计的影响，以样点邻域面片法向的加权求 和结果作为目标样点法向的估计结果，可有效抑制 曲面采样误差对法向估计结果的影响，实现点云法 向稳健估计。

（2）对局部同构曲面高斯映射结果的聚类分析 可获得样点的各向同性邻域面，以此作为样点法向 加权估计样本，可在保证法向估计过程稳健性的前 提下，提高法向估计精度以及尖锐特征区域样点法 向估计的准确性。

(3) 以局部样本 Delaunay 网格剖分结果估计目 标样点法向，避免了基于全局 Delaunay 网格剖分的 法向估计算法高复杂度问题，对于大规模的点云数 据，计算效率提高约 30\%。

(4) 通过调整阈值 $\varepsilon$ 可准确获取样点各向同性 邻域面, 实现样点法向准确估计, 样点分布不均匀 或点云含有复杂细节特征时，固定的阈值可能会导 致部分样点各向同性邻域面识别准确度降低，影响 法向估计精度 , 后续工作可通过分析样点邻域样点 的分布情况自适应调整阈值，进一步提高算法的精 度和效率。

\section{参 考 文 献}

[1] MURA C , WYSS G , PAJAROLA R. Robust normal estimation in unstructured $3 \mathrm{D}$ point clouds by selective normal space exploration[J]. The Visual Computer ,2018 , 34(6-8) : 961-971.

[2] GUERRERO P , KLEIMAN Y , OVSJANIKOV M , et al. PCPNET : Learning local shape properties from raw point clouds[J]. Computer Graphics Forum 2017,37(2) :75-85.

[3] AMENTA N, CHOI S, DEY T K, et al. A simple algorithm for homeomorphic surface reconstruction[J]. 
International Journal of Computational Geometry \& Applications , 2008 , 12(1) : 125-141.

[4] DEYT K, LI G, SUN J. Normal estimation for point clouds : A comparison study for a voronoi based

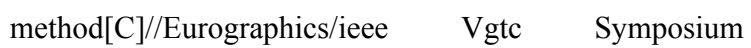
Point-Based Graphics. Piscataway : IEEE , 2008 : 39-46.

[5] MERIGOT Q , OVSJANIKOV M , GUIBAS L J. Voronoi-based curvature and feature estimation from point clouds[J]. IEEE Transactions on Visualization and Computer Graphics , 2011 , 17(6) : 743-756.

[6] CUEL L , LACHAUD J O , THIBERT B. Voronoi-based geometry estimator for 3D digital surfaces[C]// Discrete Geometry for Computer Imagery. Siena , Italy : Springer , 2014 : 134-149.

[7] HOPPE H. Surface reconstruction from unorganized points[C]//Conference on Computer Graphics and Interactive Techniques. New York : ACM , 1992 : 71-78.

[8] NURUNNABI A , BELTON D , WEST G. Robust statistical spproaches for local planar surface fitting in 3D laser scanning data[J]. Isprs Journal of Photogrammetry \& Remote Sensing , 2014 , 96(4) : 106-122.

[9] 王醒策，蔡建平，武仲科，等. 局部表面拟合的点云模 型法向估计及重定向算法 [J]. 计算机辅助设计与图形 学学报, 2015, 27(4): 614-620.

WANG Xingce, CAI Jianping, WU Zhongke, et al. Normal estimation and normal orientation for point cloud model based on improved local surface fitting[J]. Journal of Computer-Aided Design \& Computer Graphics , 2015 , 27(4) : 614-620.

[10] CAO J ,CHEN H ,ZHANG J ,et al. Normal estimation via shifted neighborhood for point cloud[J]. Journal of Computational and Applied Mathematics , 2018 , 329 : 57-67.

[11］孙殿柱, 郭洪帅, 李延瑞, 等. 基于局部泊松曲面重建 的点云刚性配准方法 [J]. 机械工程学报, 2018, 54(15) : 141-149.

SUN Dianzhu ,GUO Hongshuai ,LI Yanrui ,et al. Method of rigid registration based on poisson reconstruction of local sample points[J]. Journal of Mechanical Engineering , 2018 , 54(15) : 141-149.

[12] CHEN Y , GAO J , WEN H, et al. Estimation normal vector of triangular mesh vertex by angle and centroid weights and its application[J]. Telkomnika Indonesian Journal of Electrical Engineering ,2013,11(4) :1841-1848.

[13] MANMI K. New weights for estimating normal surface in the triangular mesh[J]. Eurasian Journal of Science and
Engineering , $2017,3(2): 19-23$.

[14] 高健，陈岳坪，邓海祥，等. 复杂曲面零件加工精度的 原位检测误差补偿方法 $[\mathrm{J}]$. 机械工程学报，2013， 49(19) : 133-143.

GAO Jian ,CHEN Yueping ,DENG Haixiang ,et al. In-situ inspection error compensation for machining accuracy improvement of complex components[J]. Journal of Mechanical Engineering , 2013 , 49(19) : 133-142.

[15］孙殿柱，魏亮，李延瑞，等. 基于局部样本增益优化的 $\alpha$-shape 曲面拓扑重建 $[\mathrm{J}]$. 机械工程学报 ,2016,52(3) : 136-142.

SUN Dianzhu, WEI Liang, LI Yanrui, et al. Surface reconstruction with $\alpha$-shape based on optimization of surface local sample[J]. Journal of Mechanical Engineering , 2016 , 52(3) : 136-142.

[16] CHENG S W , DEY T K , SHEWCHUK J R. Delaunay mesh generation $[\mathrm{J}]$. Computational Mathematics \& Mathematical Physics , 2013 , 50(1) : 38-53.

[17] BROUMI S , BAKAL A, TALEA M , et al. Applying dijkstra algorithm for solving neutrosophic shortest path problem[C]//nternational Conference on Advanced Mechatronic Systems. Melbourne :IEEE ,2017 :412-416.

[18] 李延瑞, 孙殿柱, 张英杰, 等. 曲面边界样点逆向均值 漂移识别 [J]. 计算机集成制造系统，2015，21(7)： 1719-1724.

LI Yanrui ,SUN Dianzhu ,ZHANG Yingjie ,et al. Reverse mean shift detection algorithm for boundary points of surface[J]. Computer Integrated Manufacturing Systems , 2015 , 21(7) : 1719-1724.

[19] 张雨禾, 耿国华, 魏潇然, 等. 保留几何特征的散乱点 云简化算法 $[\mathrm{J}]$. 计算机辅助设计与图形学学报，2016, 28(9) : 1420-1427.

ZHANG Yuhe ,GENG Guohua ,WEI Xiaoran ,et al. Point clouds simplification with geometric feature reservation[J]. Journal of Computer-Aided Design \& Computer Graphics , 2016 , 28(9) : 1420-1427.

[20] 孙殿柱, 白银来, 李延瑞, 等. 散乱点集拓扑邻域均值 逆向漂移查询算法 [J]. 机械工程学报, 2015, 51(1): 182-187.

SUN Dianzhu ,BAI Yinlai ,LI Yanrui ,et al. Mean reverse shift query algorithm for topological neighbors of scattered point-cloud[J]. Journal of Mechanical Engineering , 2015 , 51(1) : 182-187.

[21] DEMARSIN K , VANDERSTRAETEN D , VOLODINE $\mathrm{T}$, et al. Detection of closed sharp edges in point clouds using normal estimation and graph theory[J]. 
Computer-Aided Design , 2007 , 39(4) : 276-283.

作者简介：孙殿柱(通信作者), 男, 1956 年出生, 博士, 教授, 博士研 究生导师。主要研究方向为数字化设计与制造、逆向工程。

E-mail : dianzhus@sdut.edu.cn

梁增凯, 男, 1992 年出生, 硕士研究生。主要研究方向为数字化设计与 制造、逆向工程。

E-mail : zengkai27@gmail.com
薄志成, 男, 1991 年出生, 硕士研究生。主要研究方向为数字化设计与 制造、逆向工程。

E-mail : bozhicheng91@gmail.com

李延瑞, 男, 1979 年出生, 博士研究生。主要研究方向为数字化设计与 制造、逆向工程。

E-mail : liyanrui.m2@gmail.com

沈江华, 男, 1995 年出生, 硕士研究生。主要研究方向为数字化设计与 制造、逆向工程。

E-mail : jianghuash@126.com 